Revista de MATEMÁticA: TeORÍA y APliCACIONEs 2016 23(2) : 489-506

CIMPA - UCR ISSN: 1409-2433 (PRINT), 2215-3373 (ONLINE)

\title{
ANÁLISIS DE UN MODELO PLANTA-HERBÍVORO APLICADO A LA INTERACCIÓN GRAMÍNEA-BOVINO
}

\section{ANALYSIS OF A PLANT-HERBIVOROUS MODEL APPLIED TO GRASS-BOVINE INTERACTION}

\author{
JHOANA P. ROMERO L.* ${ }^{*}$ EDUARDO IBARGÜEN ${ }^{\dagger}$ \\ Alejandra Pulgarín DEyber CORdero $^{\ddagger}$ \\ INGRID P. CASTAÑO
}

Received: 20 Aug 2014; Revised: 28 Aug 2015;

Accepted: 20 Feb 2016

*Est. de Doctorado, Instituto de Matemáticas, Universidad de Antioquia, Medellín, Colombia. E-Mail: jpatirom3@gmail.com

${ }^{\dagger}$ Departamento de Matemáticas y Estadística, Facultad de Ciencias, Universidad de Nariño, Pasto, Colombia. E-Mail: edbargun@gmail.com

${ }^{\ddagger}$ Facultad de Ciencias Básicas, Universidad del Quindío, Armenia-Colombia. E-Mail: ampulgarin@uniquindio.edu.co

${ }^{\S}$ Facultad de Ciencias Agrarias, Universidad de Antioquia, Medellín,Colombia. E-Mail: decosoldier.1@hotmail.com

『Misma dirección que/Same address as: D. Cordero. E-Mail: ipcc-2804@ hotmail.com 


\title{
Resumen
}

En este trabajo se describe una dinámica de interacción interespecífica gramínea-bovino a través de un modelo presa-predador con una respuesta funcional Holling tipo II. El análisis cualitativo revela la existencia de una bifurcación de Hopf supercrítica con parámetro de bifurcación decreciente que se correlaciona con tres equilibrios: uno inestable donde no existen especies, otro con solo gramínea y uno donde coexisten ambas especies. Estos resultados, validados con datos estadísticos de investigaciones realizadas en la región Caribe y valles interandinos de Colombia, muestran la relevancia de un consumo de gramínea sostenible por parte de los bovinos.

Palabras clave: gramínea; bovinos; bifurcación de Hopf; modelo presa-predador.

\begin{abstract}
In this work we describe an interspecific interaction dynamics between grass and bovines through a predator-prey model with a functional response of Holling tipe II. Qualitative analysis reveals the existence of a supercritical Hopf bifurcation with decreasing bifurcation parameter correlated with three equilibrium solutions: the trivial equilibrium without species, the equilibrium only with grass and the equilibrium of coexistence between the both species. These results were validated with statistical data from research conducted in the Caribbean region and interandinos valleys of Colombia. They show the relevance of grass sustainable consumption by bovines.
\end{abstract}

Keywords: grass; bovines; Hopf bifurcation; prey-predator model.

Mathematics Subject Classification: 34D23, 93D20, 65L05.

\section{Introducción}

Las plantas al ser seres vivos autótrofos, es decir, que toman del medio sustancias inorgánicas y energía solar para transformarla en materia orgánica (glucosa y almidones) y, al formar la base de la cadena trófica, presentan frecuentes interacciones con otros seres vivos de su entorno, entre tales interacciones se tienen: planta-planta, planta-hongo, planta-animal, planta-microorganismo [13]; cada una de éstas genera beneficio o perjuicio en dichas especies. Por ejemplo, en la interacción planta-planta la cercanía con la cual ambas especies se encuentran ubicadas, ha sido considerada como sinónimo de competencia por los recursos limitantes, tales como luz, agua y nutrientes; en el caso de especies arbustivas y plántulas leñosas quienes crecen juntas, la competencia podría derivarse del solapamiento en el uso de los recursos edáficos al situar ambas las raíces 
en el mismo volumen de suelo, en tal caso unas se ven beneficiadas y otras no tanto. Cuando se beneficia un solo individuo se considera que la interacción entre dichas especies es negativa [20], por el contrario cuando se presentan beneficios para ambas partes la competencia se considera positiva, como en el caso de árboles-pastos [6]. La interacción planta-hongo se considera positiva para ambas especies, tal es el caso de las micorrizas, las cuales presentan una sociedad que genera ganancia para ambas especies involucradas puesto que la planta recibe del hongo nutrientes, minerales y agua, y el hongo a su vez, obtiene de la planta hidratos de carbono y vitaminas que él, por sí mismo, es incapaz de sintetizar. La interacción planta-microorganismo se considera al igual que la interacción anterior, positiva para ambas especies; un ejemplo de esta relación es la rizósfera, la cual es el lugar de destino de carbohidratos producto de la fotosíntesis $y$, que las plantas exudan por sus raíces para proveer energía a los microorganismos, quienes en retribución protegen a las raíces de organismos patógenos y además, solubilizan minerales haciéndolos más asimilables para la planta. En la interacción planta-animal se pueden presentar dos casos: competencia positiva y negativa. En el primer caso, las dos especies se ven beneficiadas por dicha interacción (mutualismo), mientras que en el segundo caso solamente una especie se ve beneficiada (presa-depredador). Un caso particular de este último sistema, es la interacción planta-herbívoro, en el cual el daño sufrido por la planta como consecuencia de la acción de los herbívoros, puede ser nulo o llegar la defoliación total y posterior muerte. En la teoría, el primero hace referencia a un sistema no interactivo (el herbívoro no afecta la tasa de renovación de la vegetación), mientras que el segundo hace referencia a un sistema interactivo (el herbívoro afecta la tasa de renovación de la vegetación), aunque cabe resaltar que los herbívoros pueden tener efectos positivos sobre las plantas si los tejidos vegetales que consumen incluyen órganos reproductores tales como como semillas [21]. Un ejemplo de un sistema planta-herbívoro, es la interacción que se da entre la gramínea y el bovino, en la cual, la existencia de incompatibilidad entre estas dos especies ha establecido una marcada degradación de pastos nativos, en especial en zonas de altas temperaturas como en la gran parte de la subregión del Bajo Cauca antioqueño y el sur de Córdoba en Colombia, en donde las altas radiaciones y la ganadería extensiva de la mano con el mal manejo que algunos ganaderos dan al pastoreo, conducen a deteriorar los pastizales de la zona causando de cierta manera una desertificación [12]. La problemática mencionada anteriormente, ha sido abordada desde distintos enfoques por la comunidad científica. Desde el enfoque matemático, se han desarrollado investigaciones a nivel internacional, nacional y local, que conducen a aportar a la solución del fenómeno del sobrepastoreo. A nivel internacional, Agarwal y Mishra [1], plantean y analizan un 
modelo matemático en ecuaciones diferenciales parciales para modelar el efecto del sobrepastoreo en una pradera boscosa; el modelo es analizado utilizando la teoría de la estabilidad de Lyapunov. Martínez [14], plantea un estudio de la desertificación por sobrepastoreo mediante un modelo de simulación dinámica. Da Silveria y Mendoza [15], proponen una estrategia de gestión llamada política de umbral mediante un modelo matemático de interacción planta-agua. Algunos modelos usan respuesta funcional Holling tipo II $[22,7]$ para sistemas presadepredador. Este trabajo se concentra en analizar matemáticamente un sistema planta-herbívoro; el contacto entre las dos especies es modelado a través de una respuesta funcional de Holling tipo II, al ser una de las más frecuentes en la naturaleza [5]. Las simulaciones numéricas se realizan usando datos suministrados por estudios realizados en parte de la Región Caribe y Valles Interandinos (Colombia).

\section{El modelo}

En el siguiente modelo las variables $x(t)$ e $y(t)$ representan las poblaciones de gramínea y bovinos en un tiempo $t$, respectivamente. Se supone que la gramínea presenta crecimiento logístico con una capacidad de carga constante $K$ interpretado como el máximo producto alimenticio por hectárea o medida de cantidad que depende del ambiente natural, de la especie de gramínea y manejo que se le de a ésta [9]. La gramínea se ve disminuida por contacto con el bovino a través de una respuesta funcional de Holling tipo II; dicho contacto permite el aumento de la población de bovinos proporcionalmente a una tasa $\beta$ de transformación de biomasa vegetal en biomasa animal (tanto en crecimiento como en reproducción); esta tasa puede interpretarse también como una tasa de aprovechamiento de la gramínea consumida por el bovino. A su vez la población de bovinos disminuye proporcionalmente a una tasa $\alpha \beta$ definida como la tasa de saciedad de los bovinos (que por lo general ocurre cuando el bovino ya está listo para el sacrificio o la venta).

Con las anteriores hipótesis se obtiene el siguiente sistema de ecuaciones diferenciales ordinarias:

$$
\begin{aligned}
& \frac{d x}{d t}=x\left(1-\frac{x}{K}\right)-g(x) y \\
& \frac{d y}{d t}=-\alpha \beta y+\beta g(x) y,
\end{aligned}
$$

donde

$$
g(x)=\frac{x}{1+x}
$$


La región de interés biológico para las soluciones del sistema (1), viene dada por el siguiente conjunto:

$$
\Omega=\left\{(x, y) \in \mathbb{R}^{2}: 0 \leq x \leq K, y \geq 0, \lim _{t \rightarrow \infty} e^{\beta \int_{0}^{t}(-\alpha+g(x(\tau))) d \tau} \in(0, \infty)\right\} .
$$

En la siguiente proposición se prueba que el sistema (1) está bien planteado en el sentido que soluciones con condiciones iniciales en $\Omega$ permanecen allí para todo $t \geq 0$.

Proposición 2.1 El conjunto $\Omega$ definido en (3) es positivamente invariante para las soluciones del sistema de ecuaciones diferenciales ordinarias (1).

Demostración 2.2 A partir de la primera ecuación de (1) se obtiene la desigualdad

$$
\frac{d x}{d t} \leq x\left(1-\frac{x}{K}\right)
$$

cuya solución satisface $0 \leq x(t) \leq K$. Por otro lado, la solución de la segunda ecuación de (1) es

$$
y(t)=y(0) e^{\beta \int_{0}^{t}(-\alpha+g(x(\tau)) d \tau},
$$

lo cual implica que $\lim _{t \rightarrow \infty} y(t)$ existe si y solo si

$$
\lim _{t \rightarrow \infty} e^{\beta \int_{0}^{t}(-\alpha+g(x(\tau))) d \tau} \in(0, \infty),
$$

existe. Finalmente, se verifica fácilmente que el campo vectorial definido por (1) sobre $\partial \Omega$ no apunta hacia el exterior de $\Omega$. Por lo tanto, cualquier solución de (1) que inicie en $\Omega$ permanecerá allí para todo $t \geq 0$.

\section{Soluciones de equilibrio}

Los estados de equilibrio del sistema (1) están dados por las soluciones del siguiente sistema algebraico

$$
\begin{aligned}
x\left(1-\frac{x}{K}\right)-g(x) y & =0 \\
-\alpha \beta y+\beta g(x) y & =0 .
\end{aligned}
$$

Reemplazando $g(x)$ definido en (2) en el sistema (4) se obtiene

$$
\begin{aligned}
x\left[\left(1-\frac{x}{K}\right)-\frac{y}{1+x}\right] & =0 \\
\beta y\left[-\alpha+\frac{x}{1+x}\right] & =0 .
\end{aligned}
$$


Obsérvese que cuando $y=0$, la primera ecuación de (5) se reduce a

$$
x\left(1-\frac{x}{K}\right)=0 .
$$

Las soluciones de (6) están dadas por $x=0$ y $x=K$ lo cual implica que

$$
P_{0}=(0,0) \text { y } P_{1}=(K, 0),
$$

son equilibrios de (1). Cuando $y \neq 0$, de la segunda ecuación del sistema (5) se obtiene

$$
x=\frac{\alpha}{1-\alpha},
$$

lo cual implica que $0<\alpha<1$. Por otro lado, reemplazando (8) en la primera ecuación de (5) y despejando la variable $y$ se obtiene

$$
y=\frac{K-(K+1) \alpha}{K(1-\alpha)^{2}}
$$

lo cual implica la existencia del tercer equilibrio

$$
P_{2}=\left(\frac{\alpha}{1-\alpha}, \frac{K-(K+1) \alpha}{K(1-\alpha)^{2}}\right) .
$$

Nótese que $P_{2}$ tiene sentido biológico si y solo si

$$
\alpha<\frac{K}{K+1} .
$$

Los resultados anteriores sobre existencia de soluciones de equilibrio del sistema (1) se resumen en la siguiente proposición.

Proposición 3.1 Siempre existen las soluciones de equilibrio $P_{0}=(0,0)$ y $P_{1}=(K, 0)$ del sistema (1). Si se satisface la condición (10), además de $P_{0}$ y $P_{1}$ existe una tercera solución de equilibrio $P_{2}$ definida en (9).

\section{Análisis de estabilidad local}

En esta sección se determina la estabilidad asintótica local de las soluciones de equilibrio del sistema (1). Para este fin, se inicia analizando la estabilidad local del equilibrio trivial $P_{0}=(0,0)$ en la región de interés $\Omega$. La linealización del sistema (1) alrededor de un punto de equilibrio $P$ está dada por

$$
J(P)=\left(\begin{array}{cc}
1-\frac{2 x}{K}-\frac{y}{(1+x)^{2}} & -\frac{x}{1+x} \\
\frac{\beta y}{(1+x)^{2}} & \beta\left(\frac{x}{x+1}-\alpha\right)
\end{array}\right) .
$$


A partir de (11) se tiene que la matriz jacobiana $J$ evaluada en $P_{0}$ es

$$
J\left(P_{0}\right)=\left[\begin{array}{cc}
1 & 0 \\
0 & -\alpha \beta
\end{array}\right] .
$$

Obsérvese que los valores propios de (12) están dados por $\lambda_{1}=1$ y $\lambda_{2}=-\alpha \beta$ lo cual implica que $P_{0}=(0,0)$ es inestable, más aún, es un punto de silla. El resultado anterior se resume en la siguiente proposición.

Proposición 4.1 El equilibrio trivial $P_{0}$ es inestable.

Ahora, se determinará la estabilidad del equilibrio $P_{1}$. Para este fin, obsérvese que la matriz $J$ evaluada en $P_{1}$ está dado por

$$
J\left(P_{1}\right)=\left(\begin{array}{cc}
-1 & -\frac{K}{1+K} \\
0 & \beta\left(\frac{K}{K+1}-\alpha\right)
\end{array}\right),
$$

cuyos valores propios son

$$
\lambda_{3}=-1 \quad \text { y } \quad \lambda_{4}=\beta\left(\frac{K}{K+1}-\alpha\right) .
$$

Por lo tanto $\lambda_{4}<0$ sí y solo si

$$
\alpha>\frac{K}{K+1} .
$$

El resultado anterior se resume en la siguiente proposición.

Proposición 4.2 Si se satisface (14) el equilibrio trivial $P_{1}$ es local y asintóticamente estable en $\Omega$.

Finalmente, se determinarán las condiciones para las cuales el equilibrio $P_{2}$ definido en (9) es estable en $\Omega$. Obsérvese que para $x \neq 0$ y $y \neq 0$ la ecuaciones de equilibrio (5) se reducen a

$$
\begin{aligned}
1-\frac{x}{K}-\frac{y}{1+x} & =0 \\
-\alpha+\frac{x}{1+x} & =0 .
\end{aligned}
$$

Reemplazando (15) en (11) y simplificando la matriz Jacobiana se reescribe como

$$
J(P)=\left(\begin{array}{cc}
x\left(\frac{y}{(1+x)^{2}}-\frac{1}{K}\right) & -\frac{x}{1+x} \\
\frac{\beta y}{(1+x)^{2}} & 0
\end{array}\right) .
$$


En este caso, la matriz jacobiana $J$ evaluada en $P_{2}$ se reduce a

$$
J\left(P_{2}\right)=\left(\begin{array}{cc}
\frac{\alpha[K-1-(K+1) \alpha]}{(1-\alpha) K} & -\alpha \\
\frac{\beta[K-(K+1) \alpha]}{K} & 0
\end{array}\right) .
$$

La ecuación característica de la matriz definida en (17) es

$$
\lambda^{2}-\operatorname{Tra} J\left(P_{2}\right) \lambda+\operatorname{Det} J\left(P_{2}\right)=0,
$$

donde

$$
\operatorname{Tra} J\left(P_{2}\right)=\frac{\alpha[(K+1) \alpha+1-K]}{(1-\alpha) K} \quad \text { y } \quad \operatorname{Det} J\left(P_{2}\right)=\frac{\alpha \beta[K-(K+1) \alpha]}{K} .
$$

A partir de (10) se verifica que $\operatorname{Det} J\left(P_{2}\right)>0$. Por otro lado, $\operatorname{Tra} J\left(P_{2}\right)>0$ si y solo si se satisface la siguiente desigualdad

$$
\alpha>\frac{K-1}{K+1} .
$$

En consecuencia, basta con que se satisfaga (18) para que $P_{2}$ sea local y asintóticamente estable. El resultado anterior se resume en la siguiente proposición.

Proposición 4.3 Si se satisface (18) entonces $P_{2}$ es localmente asintóticamente estable en $\Omega$.

La tabla 1 resume el comportamiento dinámico del sistema (1).

Tabla 1: Condiciones de existencia y estabilidad de los estados de equilibrio del sistema (1).

\begin{tabular}{c|cc}
\hline \hline Equilibrio & Existencia & Estabilidad \\
\hline$P_{0}$ & Siempre existe & Inestable \\
$P_{1}$ & Siempre existe & $\alpha>\frac{K}{K+1}$ \\
$P_{2}$ & $\alpha<\frac{K}{K+1}$ & $\alpha>\frac{K-1}{K+1}$ \\
\hline \hline
\end{tabular}

\subsection{Análisis de bifurcación y estabilidad global}

En esta sección se verifica la existencia de una bifurcación de Hopf supercrítica para

$$
\alpha=\alpha_{0}=\frac{K-1}{K+1}
$$


Obsérvese que para $\alpha=\alpha_{0}$ el equilibrio $P_{2}$ se reescribe como

$$
P_{2,0}=\left(\frac{K-1}{2}, \frac{(K+1)^{2}}{4 K}\right) .
$$

En este caso la matriz jacobiana se reduce a

$$
J\left(P_{2,0}\right)=\left(\begin{array}{cc}
0 & -\alpha \\
\frac{\beta}{K} & 0
\end{array}\right),
$$

cuyos valores propios son $\pm i \sqrt{\alpha \beta / K}$, lo cual implica que $P_{2}$ se transforma en el equilibrio no hiperbólico $P_{2,0}$. Lo anterior abre la posibilidad de la existencia de una bifurcacion de Hopf. Con el propósito de verificar las hipótesis del Teorema de Hopf, se traslada el equilibrio $P_{2,0}$ definido en (19) al orígen, por medio del cambio de coordenadas $u=x-a$ y $w=y-b$, donde

$$
\begin{aligned}
& a=\frac{K-1}{2} \\
& b=\frac{(K+1)^{2}}{4 K} .
\end{aligned}
$$

En las nuevas coordenadas $(u, w)$ el sistema (1) se reescribe como

$$
\begin{aligned}
\dot{u} & =(u+a)\left(1-\frac{u+a}{K}\right)-\frac{(u+a)(w+b)}{(1+u+a)}=\varphi(u, w) \\
\dot{w} & =-\alpha \beta(w+b)+\beta \frac{(u+a)(w+b)}{(1+u+a)}=\psi(u, w) .
\end{aligned}
$$

La linealización del sistema (21) está dada por la matriz jacobiana

$$
J(u, w)=\left[\begin{array}{cc}
1-\frac{2(u+a)}{K}-\frac{(w+b)}{(1+u+a)^{2}} & -\frac{u+a}{1+u+a} \\
\beta \frac{(w+b)}{(1+u+a)^{2}} & -\alpha \beta+\beta \frac{(u+a)}{1+u+a}
\end{array}\right] .
$$

Evaluando la matriz (22) en el orígen se tiene

$$
J(0,0)=\left[\begin{array}{cc}
1-\frac{2 a}{K}-\frac{b}{(1+a)^{2}} & -\frac{a}{1+a} \\
\beta \frac{b}{(1+a)^{2}} & -\alpha \beta+\beta \frac{a}{1+a}
\end{array}\right] .
$$

Reemplazando los valores de $a$ y $b$ definidos en (20), la matriz anterior se reduce a

$$
J(0,0)=\left[\begin{array}{cc}
2-K+\frac{1}{K} & -\frac{K-1}{K+1} \\
\frac{\beta}{K} & 0
\end{array}\right],
$$


o equivalentemente

$$
J(0,0)=\left[\begin{array}{cc}
2-\frac{(1+K)^{2} \alpha}{K} & -\alpha \\
\frac{\beta}{K} & 0
\end{array}\right] .
$$

La ecuación característica de la matriz (4.1) es

$$
\lambda^{2}-\operatorname{Tra}(J(0,0)) \lambda+\operatorname{Det}(J(0,0))=0,
$$

donde

$$
\begin{aligned}
\operatorname{Tra}(J(0,0)) & =2-\frac{(1+K)^{2} \alpha}{K} \\
\operatorname{Det}(J(0,0)) & =\frac{\alpha \beta}{K} .
\end{aligned}
$$

Dado que $\operatorname{Det}(J(0,0))>0$, entonces los valores propios de la matriz (23) son imaginarios puros sí y solo si

$$
\mu(\alpha)=\operatorname{tra}(J(0,0))=2-\frac{(1+K)^{2} \alpha}{K}=0 .
$$

Despejando $\alpha$ se tiene que

$$
\alpha=\frac{2 K}{(K+1)^{2}}=\alpha_{0,0}
$$

Dado que

$$
\mu^{\prime}\left(\alpha_{0,0}\right)=-\frac{(1+K)^{2}}{K} \neq 0,
$$

se concluye que el sistema (21) presenta una bifurcación de Hopf alrededor del orígen, y por lo tanto el sistema en coordenadas (1) presenta bifurcación de Hopf alrededor del equilibrio de coexistencia $P_{2,0}$. Por otro lado, siguiendo un procedimiento similar al realizado en el Ejemplo 3.1 (Hopf bifurcation in a predatorprey model, página 101) del libro de Yuri Kuznetsov [8] se verifica que el primer coeficiente de Lyupunov $l_{1}(0)$ está dado por la expresión

$$
\begin{aligned}
l_{1}(0)= & -\frac{\alpha^{2}}{2 w_{0}^{2}(1+\alpha \gamma)}\left[\frac{8 \beta}{K(K+1)^{2}}+\frac{4 \alpha \beta^{2}}{K}+\frac{8}{(K+1)^{2}}\right] \\
& -\frac{\alpha^{2} w_{0}}{2 w_{0}^{2}(1+\alpha \gamma)}\left(\frac{24 \alpha \beta}{K^{2}(K+1)^{2}}+\frac{32 \beta}{(K+1)^{4}}\right)
\end{aligned}
$$


donde

$$
w_{0}=\sqrt{\frac{2 \beta}{(K+1)^{2}}} \quad \text { y } \quad \gamma=\frac{\beta}{K} .
$$

Puesto que todos los términos son positivos, concluímos que $l_{1}(0)$ definido en (27) es negativo, por tanto la bifurcación de Hopf es supercrítica. Por otro lado, cuando

$$
\alpha=\alpha_{1}=\frac{K}{K+1}
$$

el equilibrio $P_{2}$ se reescribe como

$$
P_{2,1}=(K, 0)=P_{1} .
$$

En este caso la matriz jacobiana se reduce a

$$
J\left(P_{2,1}\right)=\left(\begin{array}{cc}
-1 & -\alpha_{1} \\
0 & 0
\end{array}\right)
$$

cuyos valores propios son $0 \mathrm{y}-1$, lo cual implica que $P_{2}$ se transforma en el equilibrio no hiperbólico $P_{2,1}=P_{1}$. En la Figura 1 se presenta un diagrama de bifurcación de las soluciones de equilibrio, en el cual se observa que $P_{1}$ y $P_{2}$ son inestables cuando $\alpha<\alpha_{0}$, que $P_{2}$ es el único nodo cuando $\alpha \in\left(\alpha_{0}, \alpha_{1}\right)$, mientras que $P_{1}$ es el único nodo cuando $\alpha>\alpha_{1}$. Ahora, dado que los equilibrios $P_{0}, P_{1}$ y $P_{2}$ son inestables cuando $\alpha<\alpha_{0}$, entonces a partir del Teorema de Poincaré-Bendixon se concluye la existencia de un ciclo límite estable cuando $\alpha<\alpha_{0}$. Lo anterior implica que en $\alpha=\alpha_{0}$ se presenta una bifurcaión de Hopf supercrítica decreciente: es decir, el equilibrio $P_{2}$ estable se bifurca en un ciclo límite estable cuando $\alpha=\alpha_{0}$ a medida que el parámetro $\alpha$ decrece. Finalmente, en la región $\left(\alpha_{0}, \alpha_{1}\right)$ el equilibrio $P_{2}$ es el único equilibrio estable y además no existen órbitas cerradas en, en consecuencia $P_{2}$ es globalmente estable en esta región. De manera similar, se verifica que en la regi'on $\left(\alpha_{1}, \infty\right)$ el equilibrio $P_{1}$ es globalmente estable.

\section{Simulaciones numéricas}

En esta sección se presentan algunas simulaciones numéricas y gráficas que ilustran el crecimiento de la población de bovinos alimentados con Panicum maximun (uno de los pastos más frecuentes y cultivados en la subregión del Bajo Cauca Antioqueño [3]) por un tiempo inferior o igual a 24 horas por día.

El Panicum maximun también conocido como pasto guinea, pajarita o india son plantas perennes que forman macollas, pueden alcanzar hasta $3 \mathrm{~m}$ de altura. Los tallos son erectos y ascendentes con un vena central pronunciada. $\mathrm{Su}$ 


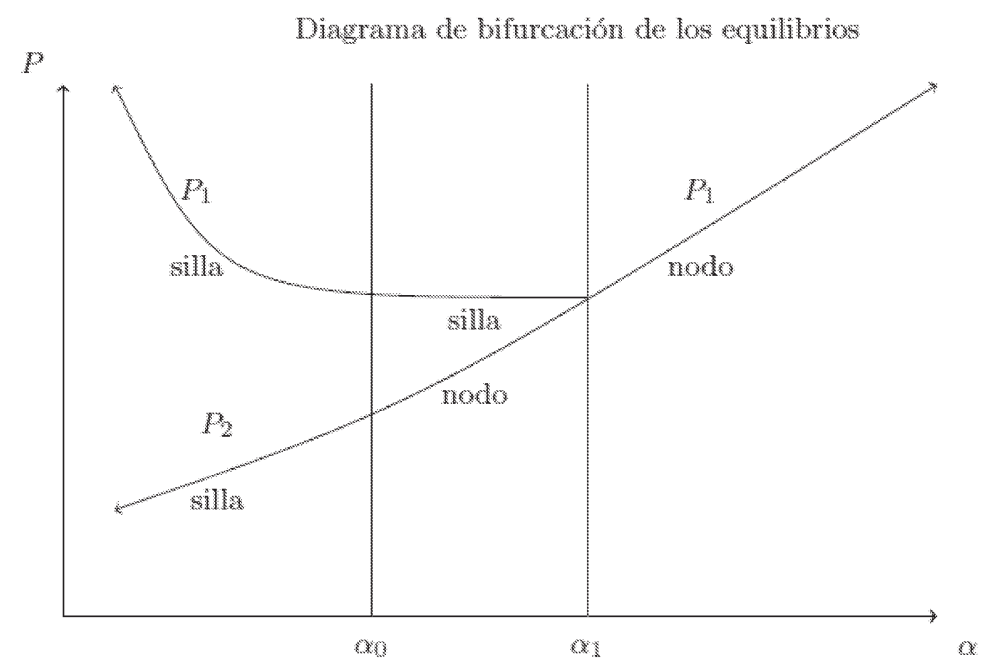

Fig. 1: Diagrama de bifurcación: Comportamiento de las soluciones de equilibrio vs el parámetro $\alpha$.

inflorescencia se presenta en forma de panoja abierta de 12 a $40 \mathrm{~cm}$ de longitud. Las raíces son fibrosas, largas y nudosas y ocasionalmente tienen rizomas, esto confiere cierta tolerancia a la sequía. Necesitan suelos de media a alta fertilidad, bien drenados con $\mathrm{pH}$ de 5 a 8 y no tolera suelos inundables, alturas entre 0 y 1500 m.s.n.m y precipitación entre $1000 \mathrm{~mm}$ y $3500 \mathrm{~mm}$ por año. Crece muy bien en temperaturas altas, aunque tienen menor tolerancia a la sequía que los Brachiarias [19]. Soportan un pastoreo intensivo pero solo con el mantenimiento de la fertilidad del suelo y responde bien a fertilización. Generalmente recomiendan retirar los animales de la pastura cuando ésta alcance 20 cm de altura [11]. En la Tabla 2 se presentan los valores de los parámetros utilizados en las simulaciones numéricas. Además, se considera que el parámetro $\alpha$ sufre pequeñas variaciones de un tiempo aproximadamente igual a 24 horas $(\alpha \in[0.9994720,0.999479])$ y la capacidad de carga $K$ del pasto guinea está medida en tonelada por hectárea de materia seca (pasto deshidratado) [3].

Tabla 2: Valores para los parámetros $K$ y $\beta$ del modelo (1).

\begin{tabular}{c|lcc}
\hline \hline Parámetros & Definición & Valor & Referencias \\
\hline$K$ & Cap. de carga del p. guinea & 3.787 & {$[3,11]$} \\
$\beta$ & Tasa de trans. de p. guinea & $443 \mathrm{gr} / \mathrm{dia}$ & {$[3,11]$} \\
$\alpha=\alpha_{0}$ & Tiempo de alimentación & 0.99947201 & Estimado \\
\hline \hline
\end{tabular}


Las gráficas de la Figura 2 fueron realizadas con diferentes valores de $\alpha$, en ellas se puede observar la aparición de las órbitas periódicas cuando $\alpha<\alpha_{0}$, el comportamiento de la espiral débil cuando $\alpha=\alpha_{0}$ y la solución de equilibrio de coexistencia cuando $\alpha>\alpha_{0}$ lo cual concuerda con los resultados teóricos.
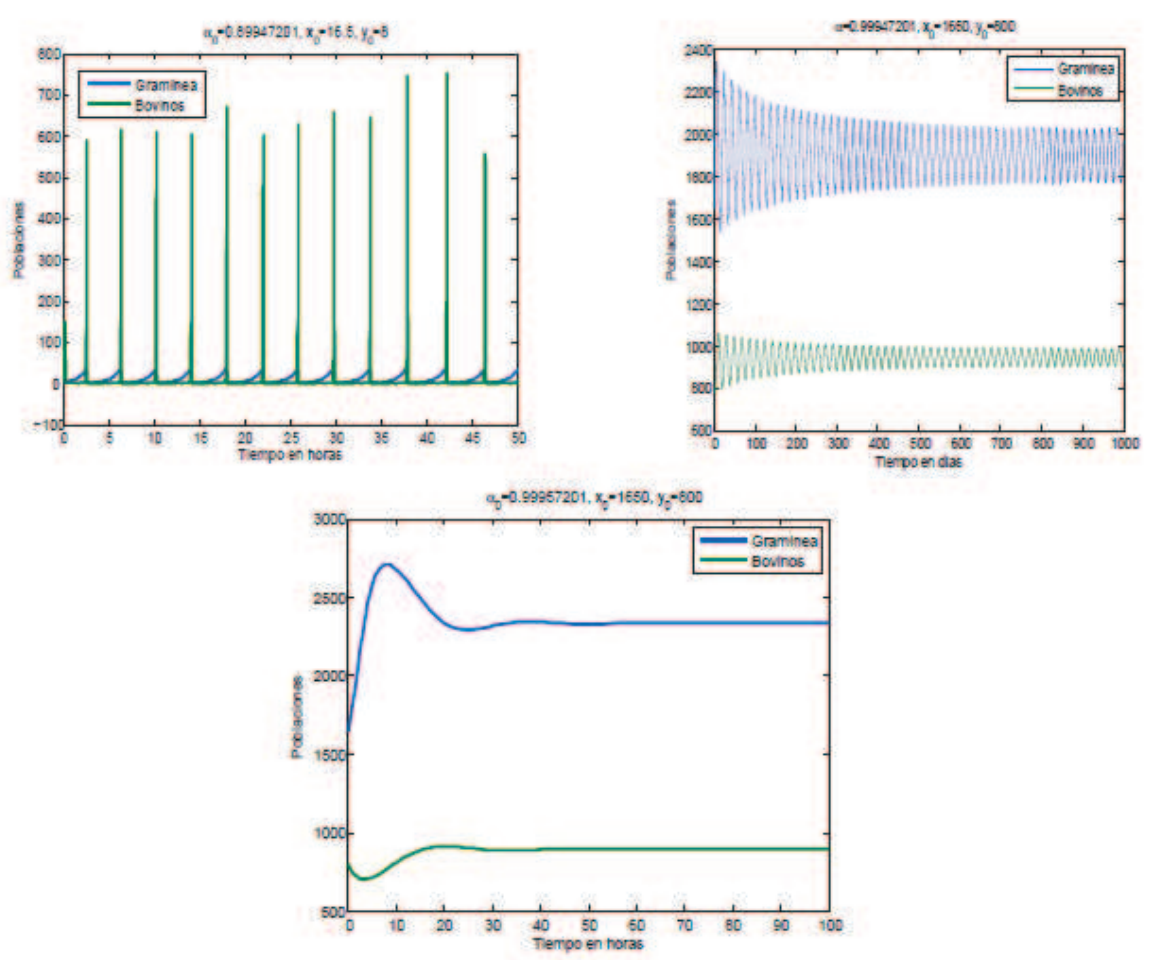

Fig. 2: Pequeñas variaciones sobre el parámetro de bifurcación $\alpha_{0}=0.99947201$.

La gráfica que aparece en la Figura 3 se realizó con lo datos de la Tabla 2, esta gráfica muestra una espiral débil, lo cual implica que ambas poblaciones buscan alcanzar su valor de coexistencia de una forma muy lenta.

Cuando los bovinos son dejados en su zona de pastoreo por un tiempo mayor que $\alpha_{0}(\alpha=0.999479)$, se genera un foco atractor (espiral) como se muestra en la Figura 4, indicando que con dicho tiempo de alimentación las poblaciones de bovinos y gramínea tienden a alcanzar su valor de coexistencia en un tiempo menor que en el anterior caso, es decir, la gramínea permanecerá constante y solo soportará una población constante de bovinos. 


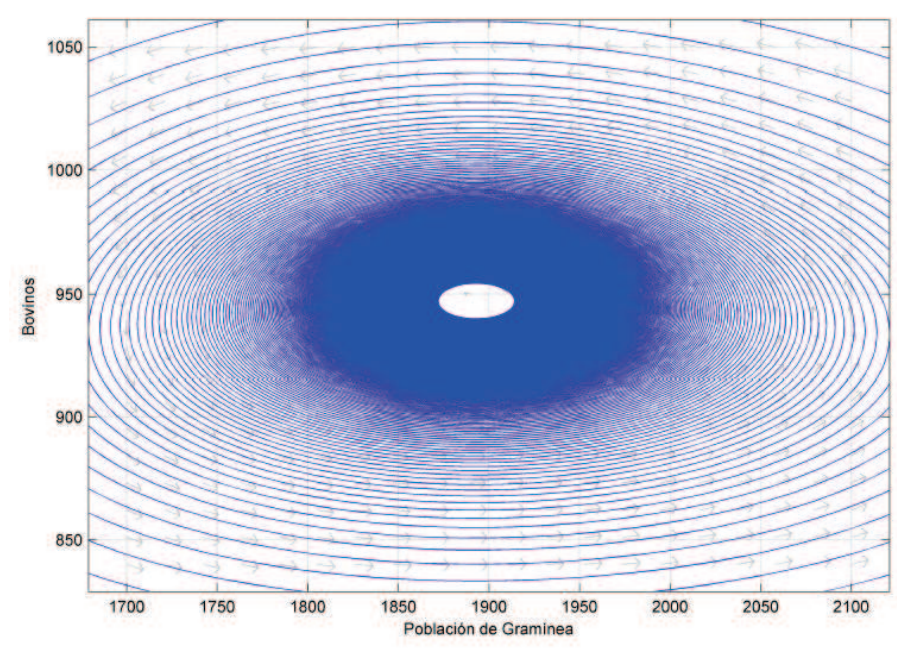

Fig. 3: Espiral Débil: Alimentación con pasto guinea por un tiempo $\alpha_{0}(24$ horas aprox.).

Finalmente, en la Figura 5 se genera un foco repulsor con ciclo límite estable (órbita), indicando que cuando los bovinos son dejados en su zona de alimentación por un tiempo menor que $\alpha_{0}(\alpha=0.9994720)$, las dos especies coexisten de manera periódica, lo cual garantiza un balance entre la gramínea y los bovinos en el sentido que una vez los bovinos consumen la suficiente gramínea o la gramínea alcanza la mínima altura que le garantice regenerarse, ellos buscan otra fuente de alimentación hasta que la gramínea alcance su altura máxima y así se repita el proceso nuevamente.

\section{Conclusiones}

En este artículo se formula un modelo matemático simple sobre la interacción planta-herbívoro, en el cual el contacto entre la gramínea (planta) y el bovino (herbívoro) es analizado mediante un modelo tipo presa-predador con una respuesta funcional de Holling tipo II (una de las más frecuentes en la naturaleza).

El análisis cualitativo revela la existencia de tres soluciones de equilibrio, el equilibrio trivial $P_{0}=(0,0)$, el equilibrio con solo gramínea $P_{1}=(K, 0)$ y el equlibrio de coexistencia entre gramínea y bovinos $P_{2}=(\alpha /(1-\alpha),[1-$ $\left.(1+1 / K) \alpha] /\left(1-\alpha^{2}\right)\right)$. Considerando $\alpha$ como el parámetro de bifurcación se verificó la existencia de una bifurcación de Hopf supercrítica en el equilibrio $P_{2}$ 


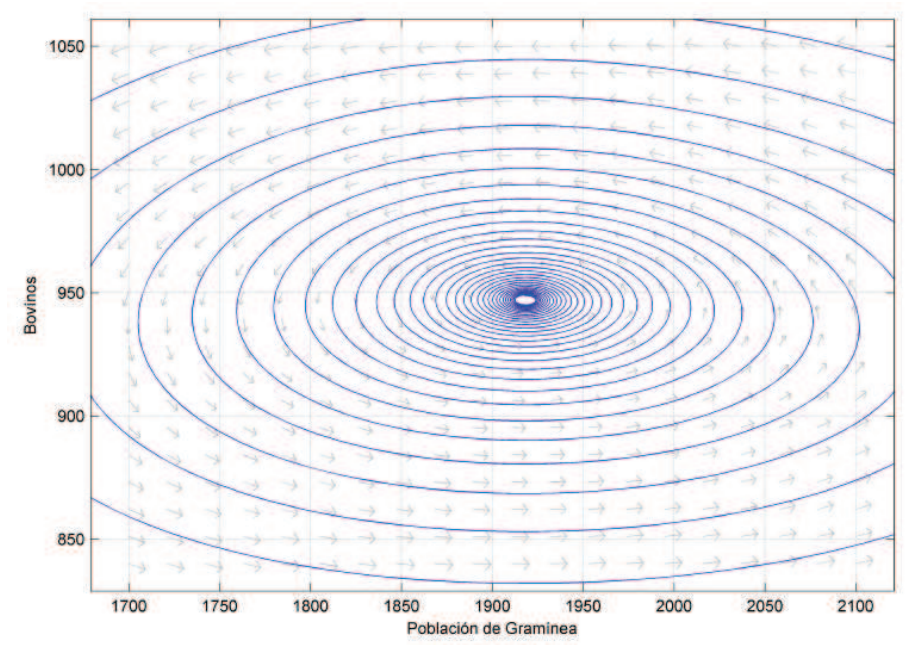

Fig. 4: Espiral: Alimentación con pasto guinea por un tiempo mayor que $\alpha_{0}$.

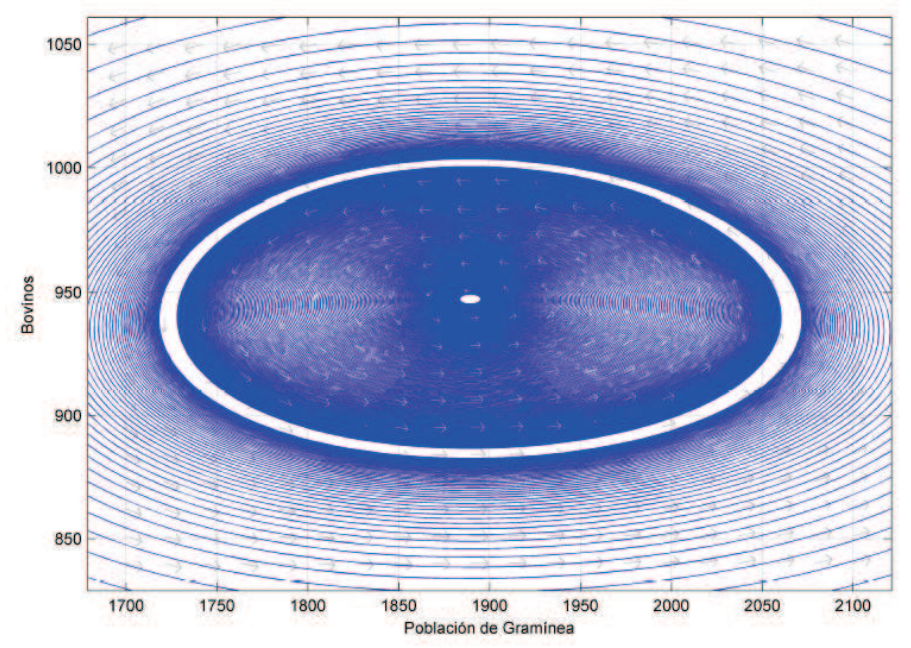

Fig. 5: Orbita: Alimentación con pasto guinea por un tiempo menor que $\alpha_{0}$. 
cuando $\alpha=\alpha_{0}=(K-1) /(K+1)$ mientras para $\alpha=\alpha_{1}=K /(K+1)$ el equilibrio de coexistencia $P_{2}$ se bifurca en el equilibrio $P_{1}$.

A partir de las simulaciones numéricas realizadas con datos de la especie de gramínea Panicum maximun establece que jornadas de alimentación del ganado bovino menores de 24 horas son las más recomendables, esto debido a que permite que la graminea se regeenre o rebrote en un tiempo prudencial. Si se supera este umbral de 24 , los resultados sugieren que hasta cierto umbral la graminea presentará capacidad para alimentar una población constante de ganado bovino hasta llegar a su capacidad de carga.

En un trabajo futuro esperamos determinar el tiempo de consumo óptimo de graminea por medio de la aplicación de teoría de control óptimo.

\section{Agradecimientos}

Jhoana Romero agradece el apoyo de la Dirección de Regionalización de la Universidad de Antioquia, E. Ibargüen agradece el apoyo recibido del proyecto número 082-16/08/2013 (VIPRI-UDENAR), D. Cordero e I. Castaño agradecen al profesor-zootecnista Juan Manuel Rojo por la supervisión e información suministrada en la investigación y A. Pulgarín agradece la colaboración del profesor Paulo César Carmona.

\section{Referencias}

[1] Agarwal, N.; Mishra, N. (2001) "A model for a forested grassland: effect or overgrazing and pollution", Indian Journal of Pure and Applied Mathematics 32(1): 613-628.

[2] Baca Carrasco, D. (2007) Análisis Paramétrico de la Bifurcación de Hopf en Sistemas Tipo Lorenz. Tesis de Maestría, Departamento de Matemáticas, Universidad de Sonora, Sonora, México.

[3] Cuesta-Muñoz, P.; Mateus Echavarria, H.; Santana R.M.; Barro Henríquez, J. (2006) "Estrategias de manejo de praderas para mejorar la productividad de la ganadería en las regiones Caribe y Valles Interandinos", in: P. Cuesta (Ed.) Producción y Utilización de Recursos Forrajeros en Sistemas de Producción Bovina en el Caribe y Valles Interandinos, Corporación Colombiana de Investigación Agropecuaria (Corpoica): 43-64.

[4] Farkas, M. (2001) Dynamical Models in Biology. Academic Press, San Diego CA. 
[5] Fernández, V.; Corley, J. (2004) "La respuesta funcional: una revisión y guía experimental”, Ecología Austral 14(1): 83-93.

[6] Gea Izquierdo, G.; Cañellas Rey de Viñas, I.; Montero González, G. (2007) "Es constante el patrón espacial y temporal de la interacción árbol-pasto en dehesas", Actas de la III Reunión sobre Sistemas Agroforestales, Cuad. Soc. Esp. Cienc. For. 22: 45-50.

[7] Ko, W.; Ryu, K. (2006) "Qualitative analysis of a predator-prey model with Holling type II functional response incorporating a prey refuge", Journal of Differential Equations 231(2): 534-550.

[8] Kuznetsov , Y.A. (1998) Elements of Applied Bifurcation Theory. Springer, New York.

[9] Lafaurie, J. (2012) Logros, Legados y Derroteros 2011-2012. FEDEGANFondo Nacional del Ganado, Bogotá.

[10] Liu, R.; Gao, J. (2010) "Permanence for a delayed discrete ratio-dependent $\mathrm{N}$-species predator-prey system with Holling-type II functional response", Mathematical and Computer Modelling, in press.

[11] Mahecha, L.; Gallego, L.A.; Peláez, F.J. (2002) "Situación actual de la ganadería de carne en Colombia y alternativas para impulsar su competitividad y sostenibilidad", Revista Colombiana de Ciencias Pecuarias 15(2): 213-225.

[12] Maldonado, G.; Velásquez, J.E. (1994) “Determinación de la capacidad de carga y ganancia de peso de bovinos en pastoreo de gramíneas nativas en Piedemonte amazónico de Colombia”, Pasturas Tropicales 16(2): 2-8.

[13] Mandujano, S. (2011) Ecología de Poblaciones Aplicada al Manejo de Fauna Silvestre . Instituto Literario de Veracruz S.C., México.

[14] Martínez, V.J. (2005) Estudio de la Desertificación por Sobrepastoreo Mediante un Modelo de Simulación Dinámica. Tesis Doctoral en Universidad Politécnica de Madrid, Madrid, España.

[15] Mendoza, M.; Bhaya, A.; Kaszkurewicz, E.; Da Silveira, M. (2006) “Onoff policy and hysteresis on-off policy control of the herbivore-vegetation dynamics in a semi-arid grazing system", Ecological Engineering 28(2): 114-123. 
506 J. ROMERO - E. IBARGÜEN - A. PULGARÍN - D. CORDERO - I. CASTAÑO

[16] Moreno-Osorio, F.; Molina Restrepo, D. (2007) Manual Técnico: Buenas Prácticas Agropecuarias en la Producción de Ganado de Doble Propósito Bajo Confinamiento, con Caña Panelera Como Parte de la Dieta. CORPOICA, Medellín, Colombia.

[17] Perko, L. (1996) Differential Equations and Dynamical Systems, Second Edition. Springer, New York.

[18] Rincón, A. (2005) “Ceba de bovinos en pasturas de Bracharia decumbens suplementados con caña de azúcar y Cratylia argente", Pasturas Tropicales 27(1): 2-12.

[19] Tropical Forages (s.f.) "Especies forrajeras multipropósito. Panicum maximum", in: http://www.tropicalforages.info/ Multiproposito/key/Multiproposito/Media/Html/

Panicum, consultado el 28-Junio-2014, 15:20.

[20] Valverde, T.; Cano, Z. (2009) Ecología y Medio Ambiente. Pearson Educación, México.

[21] Zamora, R.; García, P.; Gómez, L. (2004) “Las interacciones planta-planta y planta-animal en el contexto de la sucesión ecológica", in: F. Valladares (Ed.) Ecología del Bosque Mediterráneo en un Mundo Cambiante. Ministerio de Medio Ambiente, EGRAF S.A, Madrid, España: 371-393.

[22] Zhou, J. (2013) "Positive steady state solutions of a Leslie-Gower predatorprey model with Holling type II functional response and density-dependent diffusion", Nonlinear Analysis: Theory, Methods y Applications 82(1): $47-65$. 